Pacific Journal of Mathematics

BIRNBAUM-ORLICZ SPACES OF FUNCTIONS ON GROUPS 


\title{
BIRNBAUM-ORLICZ SPACES OF FUNCTIONS ON GROUPS
}

\author{
IRACEMA M. BUND
}

\begin{abstract}
It is natural to ask how far the theory of closed invariant subspaces for $\mathfrak{R}_{p}(G)$ can be extended to Birnbaum-Orlicz spaces $\mathfrak{R}_{A}(G)$. If $G$ is a compact group and $A$ satisfies the $\Delta_{2}$ condition for $u \geqq u_{0} \geqq 0$, the class of all closed invariant subspaces of $\mathfrak{R}_{A}(G)$ is exactly the family $\left\{\left(\mathfrak{L}_{A}\right)_{P}: P \subset \Sigma\right\}$ where $\Sigma$ is the dual object of $G$. Distinct subsets of $\Sigma$ engender distinct subspaces.
\end{abstract}

The generalization of the classical $\mathfrak{Q}_{p}$-spaces foreshadowed by $\mathrm{Z}$. W. Birnbaum in 1930 [1] was the subject of a long article by $Z$. W. Birnbaum and W. Orlicz [2]. In the next four decades their theory has been extended by many writers, among them G. Weiss [9] and W. Luxemburg who invented convenient new definitions. More recently M. Jodeit and A. Torchinsky [7] introduced a generalization of the concept of Young's function which we adopt here.

The essential introductory definitions and theorems are stated in $\S 1$; proofs may be found in [3], [8] and [9]. In $\$ 2$ we show that if $G$ is a locally compact group, the Birnbaum-Orlicz space $\mathfrak{L}_{A}(G)$ is a left Banach $\mathfrak{L}_{1}$ - module and a right Banach $\left(\mathfrak{Q}_{1} \cap \mathfrak{Q}_{1}^{\star}\right)$-module. Finally in $\$ 3$ we establish the result stated in the synopsis. Our notation is as in [4], [5] and [6].

1. Preliminaries. (1.1) A function $A$ on $[0, \infty[$ into[0, $[0]$ will be called a generalized Young's function if it is left continuous on ]0, [, $A(u) / u$ is nondecreasing for $u>0$, and $A(0)=0$. It easily follows that

(i) $A(\alpha u) \leqq \alpha A(u)$ for $0 \leqq \alpha \leqq 1$ and $0 \leqq u<\infty$.

The zero function and the function $A(u)=\infty \cdot \xi_{0, \infty[}(u)$ are trivial generalized Young's functions. Throughout the remaining of this work the letter $A$ will denote a nontrivial generalized Young's function. We also fix $a=\sup \{u: A(u)=0\}$.

A Young's function $A_{0}$ is associated to $A$ by the equality $A_{0}(u)=$ $\int_{0}^{u} A(t) / t d t$.

(1.2) Let $(X, \mathcal{M}, \mu)$ be an arbitrary measure space. The set $\mathfrak{L}_{A}(X, M, \mu)$ of all complex-valued, $\mathcal{M}$-measurable functions defined $\mu$-a.e. on $X$, such that $\int_{X} A(\alpha|f|) d \mu<\infty$ for some positive number $\alpha$ is 
called a Birnbaum-Orlicz space. Where no confusion seems possible, we will write $\mathfrak{L}_{A}(X)$ for $\mathfrak{L}_{A}(X, \mu, \mu)$.

The equality

$$
p_{A}(f)=\inf \{k \in] 0, \infty\left[: \int_{X} A(|f| / k) d \mu \leqq 1\right\}
$$

defines a nonnegative finite-valued function on $\mathfrak{L}_{A}(X)$ which is a norm in case $A$ is convex. This suggests that we define a norm on $\mathfrak{R}_{A}(X)$ by the equality $\|f\|_{A}=p_{A_{0}}(f)$. With this norm, $\mathfrak{L}_{A}(X)$ is a Banach space.

If $f \in \mathfrak{L}_{A}(G)$ the following hold:

(ii) $\|f\|_{A} \leqq p_{A}(f) \leqq 2\|f\|_{A}$;

(iii) $\int_{X} A\left(|f| / p_{A}(f)\right) d \mu \leqq 1$, provided that $p_{A}(f)>0$.

Denoting the Young's complement of $A$ by $\bar{A}$, for $f$ in $\mathfrak{L}_{A}(X)$ and $g$ in $\mathfrak{L}_{\bar{A}}(X)$ we obtain

(iv) $\int_{X}|f g| d \mu \leqq 2 p_{A}(f) p_{\bar{A}}(g)$.

If $\mu(X)$ is finite, $\mathfrak{R}_{A}(X)$ is contained in $\mathfrak{L}_{1}(X)$ and for $f \in \mathfrak{R}_{A}(X)$ we have

(v) $\|f\|_{1} \leqq\left[4 /(\bar{A})^{-1}(1 / \mu(X))\right]\|f\|_{A}$, where $(\bar{A})^{-1}$ denotes the right inverse of $\bar{A}$.

(1.3) THEOREM. Let $f$ be a complex-valued measurable function vanishing outside of a $\sigma$-finite set. Suppose that

$$
N_{A}(f)=\sup \left\{\int_{X}|f g| d \mu: g \in \mathfrak{R}_{\bar{A}}(X), p_{\bar{A}}(g) \leqq 1\right\}<\infty .
$$

Then $f \in \mathfrak{R}_{A}(X)$ and we have $\|f\|_{A} \leqq N_{A}(f)$.

(1.4) THEOREM. Let $X$ be a locally compact Hausdorff space. Let $\mu$ be a measure obtained from a nonnegative linear functional on $\mathcal{F}_{00}(X)$, and let $M$ be the $\sigma$-algebra of all $\mu$-measurable subsets of $X$. Then each function $f$ in $\mathfrak{R}_{A}(X)$ can be written as $f_{1}+f_{2}$, where $f_{1}=f \xi_{F}$ for some $\sigma$-compact set $F$, and $\left|f_{2}\right| \leqq a p_{A}(f) \mu$-a.e. on $X$. In particular, if $a=0$, then $f$ vanishes $\mu$-a.e. outside of $a \sigma$-con pact set.

\section{Birnbaum-Orlicz spaces of functions on} groups. From here on we consider spaces $\mathfrak{R}_{A}(G, \mu, \lambda)$, where $G$ is a locally compact group, $\lambda$ is a left Haar measure on $G$, and $M$ is the $\sigma$-algebra of $\lambda$-measurable subsets of $G$. We will often write $\int_{G} f d \lambda$ as $\int_{G} f(x) d x$.

Our first theorem follows easily from (20.2) in [4], and the fact that $\mathfrak{L}_{1}(G, \mathcal{M}, \max \{1,1 / \Delta\} \lambda)$ is complete. 
(2.1) THEOREM. A complex-valued measurable function $f$ belongs to $\mathfrak{R}_{1}(G) \cap \mathfrak{R}_{1}^{\star}(G)$ if and only if $\max \{1,1 / \Delta\} f \in \mathfrak{R}_{1}(G)$. The equalities and

(i) $\|f\|=\|f\|_{1}+\|(1 / \Delta) f\|_{1}$,

(ii) $\quad\|f \mid\|=\|\max \{1,1 / \Delta\} f\|_{1}$ define equivalent norms on the linear space $\mathfrak{L}_{1}(G) \cap \mathfrak{Q}_{1}^{\star}(G)$. Precisely, we have

(iii) $\quad\|f \mid\| \leqq\|f\| \leqq 2\|f\|$ for all $f \in \mathfrak{L}_{1}(G) \cap \mathfrak{L}_{1}^{\star}(G)$. With either of these two norms, $\mathfrak{R}_{1}(G) \cap \mathfrak{R}_{1}^{\star}(G)$ is a Banach space.

(2.2) THEOREM. Let $f$ be a function in $\mathfrak{L}_{A}(G)$ and let $s$ be an arbitrary element of $G$. Then the functions $f$ and $f_{s}$ belong to $\mathfrak{R}_{A}(G)$ and we have:

(i) $p_{A}\left({ }_{s} f\right)=p_{A}(f)$;

(ii) $p_{A}\left(f_{s}\right) \leqq \max \left\{1, \Delta\left(s^{-1}\right)\right\} p_{A}(f)$.

Proof. It is clear that ${ }_{s} f$ and $f_{s}$ are $\lambda$-measurable. Relations (i) and (ii) trivially become equalities if $p_{A}(f)=0$. Suppose that $p_{A}(f)>0$.

Theorem (20.1.i) in [4], and (1.2.iii) yield the inequality $p_{A}(f) \leqq$ $p_{A}(f)$, from which (i) easily follows. Using (20.1.ii) in [4], and once again (1.2.iii) we write

$$
\int_{G} A\left(\left|f_{s}\right| / p_{A}(f)\right) d \lambda \leqq \Delta\left(s^{-1}\right)
$$

which establishes (ii) in case $\Delta\left(s^{-1}\right) \leqq 1$. For $\Delta\left(s^{-1}\right)>1$, use (1) and (1.1.i).

The following result is part of (20.7) in the Russian edition of Hewitt and Ross "Abstract Harmonic Analysis", to be published.

(2.3) Lemma. Let $f$ be a $\lambda$-measurable function on $G$. The following functions are $\lambda \times \lambda$-measurable on $G \times G$ :

$$
\begin{array}{lll}
(x, y) \rightarrow f\left(x y^{-1}\right), & (x, y) \rightarrow f\left(y^{-1} x\right), & (x, y) \rightarrow f(x), \\
(x, y) \rightarrow f\left(x^{-1}\right), & (x, y) \rightarrow f(y), & (x, y) \rightarrow f\left(y^{-1}\right) .
\end{array}
$$

(2.4) THEOREM Let $f$ be a function in $\mathfrak{R}_{A}(G)$ vanishing outside of a $\sigma$-compact set $F$ and let $g$ be a function in $\mathfrak{L}_{1}(G)$. The integral

(i) $g * f(x)=\int_{G} f\left(y^{-1} x\right) g(y) d y$

exists and is finite for almost all $x$ in $G$. The function $g{ }^{*} f$ is in $\mathfrak{R}_{A}(G)$ and we have 
(ii) $\|g * f\|_{A} \leqq 4\|f\|_{A}\|g\|_{1}$.

If $g \in \mathfrak{R}_{1}(G) \cap \mathbb{R}_{1}^{\star}(G)$, the integral

(iii) $f * g(x)=\int_{G} \Delta\left(y^{-1}\right) f\left(x y^{-1}\right) g(y) d y$

exists and is finite for $\lambda$-almost all $x$ in $G$. The function $f * g$ is in $\mathfrak{L}_{A}(G)$ and we have

(iv) $\|f * g\|_{A} \leqq 4\|f\|_{A}\|g\|$, where $\|\cdot\|$ is as in (2.1.i).

Proof. We may suppose that $g$ vanishes outside of a $\sigma$-compact set $E$. Thus the function $(x, y) \rightarrow f\left(y^{-1} x\right) g(y)$ vanishes outside of the $\sigma$-compact set $(E F) \times E$.

Let $v$ be an arbitrary function in $\mathfrak{L}_{\bar{A}}(G)$. From (2.3) we know that the mapping $(x, y) \rightarrow v(x) f\left(y^{-1} x\right) g(y)$ is $\lambda \times \lambda$-measurable. Plainly this function vanishes outside of $(E F) \times E$.

Recalling (1.2.iv) and (2.2.i), we obtain

$$
\int_{G} \int_{G}\left|v(x) f\left(y^{-1} x\right) g(y)\right| d x d y
$$

$$
\leqq 2 p_{A}(f) p_{\bar{A}}(v)\|g\|_{1} .
$$

Thus we may apply (13.10) of [4] to conclude that

$$
\begin{aligned}
\int_{G} \int_{G}\left|v(x) f\left(y^{-1} x\right) g(y)\right| d y d x \\
\quad=\int_{G} \int_{G}\left|v(x) f\left(y^{-1} x\right) g(y)\right| d x d y .
\end{aligned}
$$

From (13.10) and (13.8) in [4], we see that the integral $\int_{G} v(x) f\left(y^{-1} x\right) g(y) d y$ exists and is finite for $\lambda$-almost all $x$ in $G$, and that

$$
x \rightarrow v(x) \int_{G} f\left(y^{-1} \dot{x}\right) g(y) d y .
$$

is a function in $\mathfrak{R}_{1}(G)$; in particular it is a $\lambda$-measurable function.

We define $g * f(x)$ by the equality (i), provided the integral exists, and put $g * f(x)=0$, otherwise. It is easy to see that $g * f(x)$ is finite $\lambda$ - a.e. on $G$.

In (3) we may take $v$ to be any function in $\mathfrak{S}_{00}(G)$. Recalling (11.42) in [4], we see that $g * f$ is $\lambda$-measurable. 
Consider $v$ in $\mathfrak{R}_{\bar{A}}(G)$ with $p_{\bar{A}}(v) \leqq 1$. Taking account of (1) and (2), we obtain

$$
\begin{array}{r}
\oint_{G}|v(x)(g * f)(x)| d x \leqq \int_{G} \int_{G}\left|v(x) f\left(y^{-1} x\right) g(y)\right| d y d x \\
=\int_{G} \int_{G}\left|v(x) f\left(y^{-1} x\right) g(y)\right| d x d y \leqq 2 p_{A}(f)\|g\|_{1} .
\end{array}
$$

This implies that

$$
N_{A}(g * f) \leqq 2 p_{A}(f)\|g\|_{1}
$$

Now we observe that $g * f(x)=0$ for $x$ outside of the $\sigma$ - compact set $E F$. Thus from (4) and (1.3), we conclude that $g * f \in \mathfrak{R}_{A}(G)$ and that $\|g * f\|_{A} \leqq 2 p_{A}(f)\|g\|_{1}$. Applying (1.2.ii) to this last inequality, we obtain (ii).

Next suppose that $g \in \mathfrak{R}_{1}(G) \cap \mathfrak{Q}_{1}^{\star}(G)$. Consider the function

$$
(x, y) \rightarrow v(x) f\left(x y^{-1}\right) g(y) \Delta\left(y^{-1}\right),
$$

where $v$ is an arbitrary function in $\mathfrak{l}_{\bar{A}}(G)$. As in the previous case, we see that the function (5) is $\lambda \times \lambda$-measurable and vanishes outside of the $\sigma$-compact set $(F E) \times E$. From (1.2.iv) and (2.2.ii) we obtain

$$
\int_{G}\left|v(x) f\left(x y^{-1}\right)\right| d x \leqq 2 \max \{1, \Delta(y)\} p_{A}(f) p_{\bar{A}}(v)
$$

Thus we have

$$
\begin{aligned}
& \int_{G} \int_{G}\left|v(x) f\left(x y^{-1}\right) g(y) \Delta\left(y^{-1}\right)\right| d x d y \\
& \leqq 2 p_{A}(f) p_{\bar{A}}(v) \int_{G} \max \left\{1, \Delta\left(y^{-1}\right)\right\}|g(y)| d y \\
& =2 p_{A}(f) p_{\bar{A}}(v)\|\max \{1,1 / \Delta\} g\|_{1} \\
& \leqq 2 p_{A}(f) p_{\bar{A}}(v)\|g\|,
\end{aligned}
$$

the last inequality being a consequence of (2.1.ii) and (2.1.iii).

From this point on the proof is completely analogous to that presented above for $g * f$ and we omit it. 
Theorem (2.4) serves as a lemma for the following general result.

(2.5) Theorem. Suppose that $f \in \mathfrak{L}_{A}(G)$ and $g \in \mathfrak{L}_{1}(G)$. Then the integral

(i) $g * f(x)=\int_{G} f\left(y^{-1} x\right) g(y) d y$

exists and is finite for $\lambda$-almost all $x$ in $G$. The function $g * f$ is in $\mathfrak{L}_{A}(G)$ and we have

(ii) $\|g * f\|_{A} \leqq k\|f\|_{A}\|g\|_{1}$, where $k=4$ if $a=0$ or if $G$ is $\sigma$-compact, and $k=6$ otherwise.

If $g \in \mathfrak{R}_{1}(G) \cap \mathfrak{R}_{1}^{\star}(G)$, the integral

(iii) $f * g(x)=\int_{G} \Delta\left(y^{-1}\right) g(y) d y$

exists and is finite for $\lambda$-almost all $x$ in $G$. The function $f * g$ is in $\mathfrak{R}_{A}(G)$ and we have

(iv) $\|f * g\|_{A} \leqq k\|f\|_{A}\|g\|$, where $k$ is as above and $\|\cdot\|$ is as in (2.1.i).

Proof. If $G$ is $\sigma$-compact, the assertion follows immediately from (2.4). If $a=0$, it follows from (1.4) and (2.4). Thus we may suppose that $a>0$ and that $G$ fails to be $\sigma$-compact.

Using (1.4), we may write $f=f_{1}+f_{2}$, where $f_{1}=f \xi_{F}$ for some $\sigma$-compact set $F$, and $\left|f_{2}\right| \leqq a p_{A}(f)$. It follows that

$$
\int_{G}\left|f_{2}\left(y^{-1} x\right) g(y)\right| d y \leqq a p_{A}(f)\|g\|_{1}
$$

for all $x$ in $G$, and hence that $g * f_{2}(x)$ exists and is finite for all $x$ in $G$. A short computation, in which we use (1), gives us

$$
g * f_{2}(x)\left\|g * f_{2}\right\|_{A} \leqq p_{A}\left(g * f_{2}\right) \leqq a^{-1}\left\|g * f_{2}\right\|_{\infty} \leqq 2\|f\|_{A}\|g\|_{1} \text {. }
$$

Applying (2.4.i) to $f_{1}$, we conclude that

$$
\int_{G} f_{1}\left(y^{-1} x\right) g(y) d y+\int_{G} f_{2}\left(y^{-1} x\right) g(y) d y
$$

exists and is finite for $\lambda$-almost all $x$ in $G$. Hence the same is true of $g * f(x)$.

Inequality (ii) follows from (2) and (2.4.ii) applied to $f_{1}$. The remaining assertions are similarly established.

(2.6) TheOREM. The space $\mathfrak{L}_{1}(G) \cap \mathfrak{L}_{1}^{\star}(G)$ is a Banach algebra. 
Proof. For $f$ and $g$ in $\mathfrak{L}_{1}(G) \cap \mathfrak{L}_{1}^{\star}(G)$ we obtain

$$
((1 / \Delta) g) *((1 / \Delta) f)=(1 / \Delta)(g * f) .
$$

Thus (2.1) and (2.5.i) tell us that $g * f \in \mathfrak{L}_{1}(G) \cap \mathfrak{Q}_{1}^{\star}(G)$. We use (1) to prove that $\mathfrak{L}_{1}(G) \cap \mathfrak{L}_{1}^{\star}(G)$, with the norm $\|\cdot\|$ defined in $(2.1 . \mathrm{i})$, is a normed algebra:

$$
\|g * f\| \leqq\|g\|_{1}\|f\|_{1}+\|(1 / \Delta) g\|_{1}\|(1 / \Delta) f\|_{1} \leqq\|g\|\|f\| .
$$

(2.7) THEOREM. The space $\mathfrak{L}_{A}(G)$ is a left Banach $\mathfrak{L}_{1}$-module and $a$ right Banach $\left(\mathfrak{\Omega}_{1} \cap \mathfrak{Q}_{1}^{\star}\right)$-module.

Proof. For $g$ in $\mathfrak{L}_{1}(G)$ and $f$ in $\mathfrak{L}_{A}(G),(2.5$.ii) tells us that there is a positive number $k$ such that $\|g * f\|_{A} \leqq k\|f\|_{A}\|g\|_{1}$.

Next we show that, for $f$ as above, and $g_{1}$ and $g_{2}$ in $\mathfrak{L}_{1}(G)$, we have $g_{1} *\left(g_{2} * f\right)=\left(g_{1} * g_{2}\right) * f$. Using (20.1) of [4], we obtain the equality

$$
\int_{G} f\left(v^{-1} y^{-1} x\right) g_{2}(v) d v=\int_{G} f\left(v^{-1} x\right) g_{2}\left(y^{-1} v\right) d v,
$$

which implies that

$$
g_{1} *\left(g_{2} * f\right)(x)=\int_{G} \int_{G} f\left(v^{-1} x\right) g_{2}\left(y^{-1} v\right) g_{1}(y) d v d y .
$$

By (2.5.i), $g_{1} *\left(g_{2} * f\right)$ is in $\mathfrak{R}_{A}(G)$, and hence the integral in (1) exists and is finite $\lambda$-almost everywhere in $G$. From (1.4) we know that $g_{1}$ and $g_{2}$ vanish outside of $\sigma$-compact sets $E_{1}$ and $E_{2}$, respectively. Thus the function $(v, y) \rightarrow f\left(v^{-1} x\right) g_{2}\left(y^{-1} v\right) g_{1}(y)$ vanishes outside of the $\sigma$ compact set $\left(E_{1} E_{2}\right) \times E_{1}$. By (2.3) this function is $\lambda \times \lambda$ - measurable.

We apply (13.10) in [4] to conclude that for $\lambda$-almost all $x$ in $G$ we have

$$
\begin{gathered}
g_{1} *\left(g_{2} * f\right)(x)=\int_{G} \int_{G} f\left(v^{-1} x\right) g_{2}\left(y^{-1} v\right) g_{1}(y) d y d v \\
=\int_{G} f\left(v^{-1} x\right)\left(g_{1} * g_{2}\right)(v) d v=\left(g_{1} * g_{2}\right) * f(x) .
\end{gathered}
$$

It is now clear that $\mathfrak{R}_{A}(G)$ is a left Banach $\mathfrak{R}_{1}$ - module. The proof that $\mathfrak{R}_{A}(G)$ is a right $\operatorname{Banach}\left(\mathfrak{L}_{1} \cap L_{1}^{\star}\right)$-module is similar and we omit it.

3. Closed ideals in $\mathfrak{R}_{A}(G)$ for $G$ a compact group. Throughout this section we suppose that $G$ is compact and that $\lambda(G)=1$. 
(3.1) Theorem. If $f$ and $g$ are in $\mathfrak{Q}_{A}(G)$ the equality $g * f(x)=$ $\int_{G} f\left(y^{-1} x\right) g(y) d y$ defines a function in $\mathfrak{L}_{A}(G)$. We have

$$
\|g * f\|_{A} \leqq\left(16 /(\bar{A})^{-1}(1)\right)\|f\|_{A}\|g\|_{A} \text {. }
$$

Proof. Follows from (2.5.i), (1.2.v) and (2.5.ii).

(3.2) Theorem. The Birnbaum-Orlicz space $\mathfrak{Q}_{A}(G)$ is a Banach algebra under a norm which is a positive constant times $\|\cdot\|_{A}$.

Proof. Define $n_{A}(f)=\left(16 /(\bar{A})^{-1}(1)\right)\|f\|_{A}$ and use (3.1).

(3.3) THEOREM. Suppose that $A$ satisfies the $\Delta_{2}$-condition for $u \geqq u_{0} \geqq 0$. Then the space $\mathfrak{T}(G)$ of trigonometric polynomials on $G$ is $\|\cdot\|_{A}$-dense in $L_{A}(G)$.

Proof. Our hypothesis imply that $\mathscr{C}(G)$ is $\|\cdot\|_{A}$-dense in $\mathfrak{L}_{A}(G)$ : see [3] or [8]. Theorem (27.39.ii) of [5] tells us that $\mathfrak{T}(G)$ is uniformly dense in $\mathfrak{S}(G)$, and it is easy to see that $\mathfrak{I}(G)$ is also $\|\cdot\|_{A}$ - dense in $\widetilde{S}(G)$.

(3.4) Theorem. Let $A$ be as in (3.3). Suppose that $S$ is a closed linear subspace of $\mathfrak{L}_{A}(G)$. Then $S$ is a left [right] ideal in $\mathfrak{L}_{A}(g)$ if and only if $S$ is closed under the formation of left [right] translates.

Proof. Since $G$ is unimodular, it follows from (2.1) and (2.7) that $\mathfrak{L}_{A}(G)$ is a Banach $\mathfrak{L}_{1}$ - module with respect to convolution. From (3.2) we know that $\mathfrak{L}_{A}(G)$ is a subalgebra of $\mathfrak{R}_{1}(G)$ which is a Banach algebra with the norm $n_{A}$. Taking (3.3) into account, we see that $L_{A}(G)$ has the properties stated in (38.6.a) in [5]. Thus the theorem follows immediately from (38.22.b) of [5].

(3.5) THEOREM. Let $A$ be as in (3.3). Then the class of all closed two-sided ideals in $\mathfrak{Q}_{A}(G)$ is exactly the family $\left\{\left(\mathfrak{R}_{A}\right)_{P}: P \subset \Sigma\right\}$. Distinct subsets of $\Sigma$ engender distinct ideals.

Proofs. This is a direct application of (38.7) in [5].

\section{REFERENCES}

1. Z. W. Birnbaum, Uber Approximation im Mittel, Nachrichten von der Gesellschaft der Wissenschaften zu Gottingen, Fachgruppe I, Nr. 15, (1930), 338-343.

2. Z. W. Birnbaum und W. Orlicz, Uber die Verallgemeinerung des Begriffes der zueinander konjugierten Potenzen, Studia Math., 3 (1931), 1-67. 
3. I. M. Bund, Fourier Analysis on Birnbaum-Orlicz spaces, Dissertation, University of Washington, 1973, unpublished.

4. E. Hewitt and K. A. Ross, Abstract Harmonic Analysis, Vol. I, Heidelberg, Springer-Verlag, Grundlehren der Math. Wiss., Band 115, 1963.

5. —, Abstract Harmonic Analysis, Vol. II, Heidelberg, Springer-Verlag Grundlehren der Math. Wiss, Band 152, 1970.

6. E. Hewitt and K. R. Stromberg, Real and Abstract Analysis, Heidelberg, Springer-Verlag, 1965.

7. M. Jodeit Jr. and A. Torchinsky, Inequalities for Fourier transforms, Studia Math., T. XXXVII (1971), 245-276.

8. M. A. Krasnosel'skii and Ja. B. Rutickii, Convex functions and Orlicz spaces, Groningen (The Netherlands), transl. from Russian, 1961.

9. G. Weiss, A note on Orlicz spaces, Portugaliae Math., 15 (1956), 35-47.

Received February 22, 1974. This research was supported by the Fundacão de Amparo à Pesquisa do Estado de São Paulo. This work is part of a doctoral dissertation written at the University of Washington. The author is grateful for the invaluable guidance of her research adviser, Professor Edwin Hewitt.

University of São Paulo. 



\section{PACIFIC JOURNAL OF MATHEMATICS}

\section{EDITORS}

RICHARD ARENS (Managing Editor)

University of California

Los Angeles, California 90024

\section{J. DugundJI}

Department of Mathematics University of Southern California Los Angeles, California 90007

D. Gilbarg and J. Milgram

Stanford University

Stanford, California 94305

\section{ASSOCIATE EDITORS}
E. F. BECKENBACH
B. H. NeumanN
F. WoLF
K. YoshiDA

\section{SUPPORTING INSTITUTIONS}

\author{
UNIVERSITY OF BRITISH COLUMBIA \\ CALIFORNIA INSTITUTE OF TECHNOLOGY \\ UNIVERSITY OF CALIFORNIA \\ MONTANA STATE UNIVERSITY \\ UNIVERSITY OF NEVADA \\ NEW MEXICO STATE UNIVERSITY \\ OREGON STATE UNIVERSITY \\ UNIVERSITY OF OREGON \\ OSAKA UNIVERSITY
}

\author{
UNIVERSITY OF SOUTHERN CALIFORNIA \\ STANFORD UNIVERSITY \\ UNIVERSITY OF TOKYO \\ UNIVERSITY OF UTAH \\ WASHINGTON STATE UNIVERSITY \\ UNIVERSITY OF WASHINGTON \\ AMERICAN MATHEMATICAL SOCIETY
}

The Supporting Institutions listed above contribute to the cost of publication of this Journal, but they are not owners or publishers and have no responsibility for its contents or policies.

Mathematical papers intended for publication in the Pacific Journal of Mathematics should be in typed form or offset-reproduced (not dittoed), double spaced with large margins. Underline Greek letters in red, German in green, and script in blue. The first paragraph or two must be capable of being used separately as a synopsis of the entire paper. Items of the bibliography should not be cited there unless absolutely necessary, in which case they must be identified by author and Journal, rather than by item number. Manuscripts, in duplicate, may be sent to any one of the four editors. Please classify according to the scheme of Math. Reviews, Index to Vol. 39. All other communications should be addressed to the managing editor, or Elaine Barth, University of California, Los Angeles, California, 90024.

100 reprints are provided free for each article, only if page charges have been substantially paid. Additional copies may be obtained at cost in multiples of 50 .

The Pacific Journal of Mathematics is issued monthly as of January 1966. Regular subscription rate: $\$ 72.00$ a year (6 Vols., 12 issues). Special rate: $\$ 36.00$ a year to individual members of supporting institutions.

Subscriptions, orders for back numbers, and changes of address should be sent to Pacific Journal of Mathematics, 103 Highland Boulevard, Berkeley, California, 94708.

PUBLISHED BY PACIFIC JOURNAL OF MATHEMATICS, A NON-PROFIT CORPORATION Printed at Jerusalem Academic Press, POB 2390, Jerusalem, Israel.

$$
\begin{gathered}
\text { Copyright (C) } 1975 \text { Pacific Journal of Mathematics } \\
\text { All Rights Reserved }
\end{gathered}
$$




\section{Pacific Journal of Mathematics}

\section{Vol. 58, No. $2 \quad$ April, 1975}

Zvi Artstein and John Allen Burns, Integration of compact set-valued functions . . . . . . . . . 297

Mark Benard, Characters and Schur indices of the unitary reflection group $[321]^{3} \ldots \ldots \ldots . .309$

Simeon M. Berman, A new characterization of characteristic functions of absolutely continuous

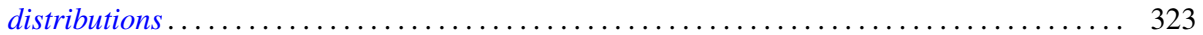

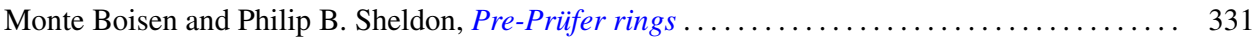

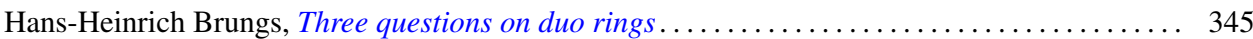

Iracema M. Bund, Birnbaum-Orlicz spaces of functions on groups................. 351

John D. Elwin and Donald R. Short, Branched immersions between 2-manifolds of higher

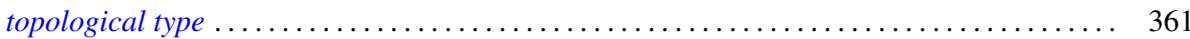

Eric Friedlander, Extension functions for rank 2, torsion free abelian groups . .......... 371

Jon Froemke and Robert Willis Quackenbush, The spectrum of an equational class of

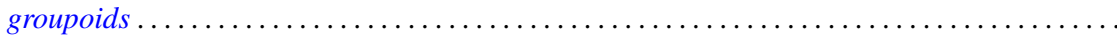

Barry J. Gardner, Radicals of supplementary semilattice sums of associative rings ...........

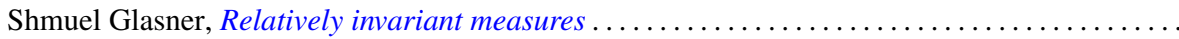

George Rudolph Gordh, Jr. and Sibe Mardesic, Characterizing local connectedness in inverse

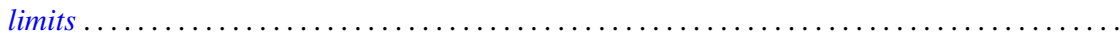

Siegfried Graf, On the existence of strong liftings in second countable topological spaces......

Stanley P. Gudder and D. Strawther, Orthogonally additive and orthogonally increasing

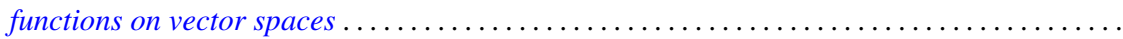

Darald Joe Hartfiel and Carlton James Maxson, A characterization of the maximal monoids and

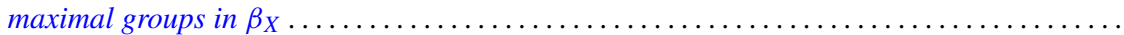

Robert E. Hartwig and S. Brent Morris, The universal flip matrix and the generalized faro-shuffle. .

William Emery Haver, Mappings between ANRs that are fine homotopy equivalences. .

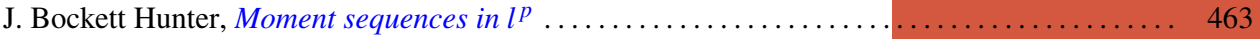

Barbara Jeffcott and William Thomas Spears, Semimodularity in the completion of a poset.... 467

Jerry Alan Johnson, A note on Banach spaces of Lipschitz functions . . . . . . . . . . . . 475

David W. Jonah and Bertram Manuel Schreiber, Transitive affine transformations on

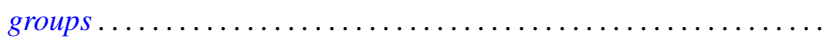

Karsten Juul, Some three-point subset properties connected with Menger's characterization of

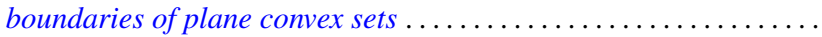

Ronald Brian Kirk, The Haar integral via non-standard analysis . . . . . . . . . . . . . 517

Justin Thomas Lloyd and William Smiley, On the group of permutations with countable

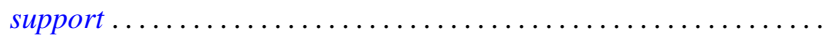

Erwin Lutwak, Dual mixed volumes .................................. 531

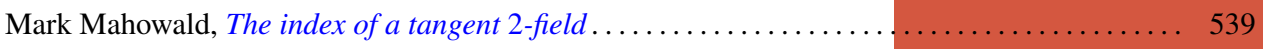

Keith Miller, Logarithmic convexity results for holomorphic semigroups . . . . . . . . . . . . 549

Paul Milnes, Extension of continuous functions on topological semigroups . . . . . . . . . . 553

Kenneth Clayton Pietz, Cauchy transforms and characteristic functions ................ 563

James Ted Rogers Jr., Whitney continua in the hyperspace $C(X) \ldots \ldots \ldots \ldots \ldots \ldots \ldots \ldots .569$

Jean-Marie G. Rolin, The inverse of a continuous additive functional . . . . . . . . . . . . 585

William Henry Ruckle, Absolutely divergent series and isomorphism of subspaces . ........ 605

Rolf Schneider, A measure of convexity for compact sets . ..................... 617

Alan Henry Schoenfeld, Continous measure-preserving maps onto Peano spaces .......... 627

V. Merriline Smith, Strongly superficial elements .......................... 643

Roger P. Ware, A note on quadratic forms over Pythagorean fields . . . . . . . . . . . . . . 651

Roger Allen Wiegand and Sylvia Wiegand, Finitely generated modules over Bezout rings . . . . 655

Martin Ziegler, A counterexample in the theory of definable automorphisms . . . . . . . . . 665 